\title{
ON ORDERED $\Lambda$-BOUNDED VARIATION
}

\author{
FRANCISZEK PRUS-WIŚNIOWSKI
}

(Communicated by R. Daniel Mauldin)

\begin{abstract}
An example is given of a continuous real function that is of ordered $\Lambda$-bounded variation but not of $\Lambda$-bounded variation. No special assumptions on $\Lambda$ are required.
\end{abstract}

Preliminaries and motivation. Let $\Lambda=\left(\lambda_{i}\right)$ be a $\Lambda$-sequence, i.e. a nonincreasing convergent-to- 0 sequence of positive numbers with $\sum \lambda_{i}=\infty$. For $m=0,1,2, \ldots$ we will denote the $\Lambda$-sequence $\left(\lambda_{m+i}\right)_{i=1}^{\infty}$ by $\Lambda_{(m)}$. Let $\langle\alpha, \beta\rangle$ be a closed interval and $x$ be a real function defined on $\langle\alpha, \beta\rangle$. For a subinterval $I=\langle s, t\rangle$ of $\langle\alpha, \beta\rangle$ we set $r(x, I)=x(t)-x(s), \quad x(I)=$ $|x(t)-x(s)|$ and $\Lambda-\operatorname{Var}(x ;\langle s, t\rangle)=\sup \sum \lambda_{i} x\left(I_{i}\right)$, where the supremum is taken over all sequences $\left(I_{i}\right)$ of nonoverlapping closed subintervals of $\langle s, t\rangle$. If $\Lambda-\operatorname{var}(x ;\langle\alpha, \beta\rangle)$ is finite then we say $x$ is of $\Lambda$-bounded variation (on $\langle\alpha, \beta\rangle)$ and write $x \in \Lambda \mathrm{BV}$.

A finite collection $\left\{I_{i}: i=1, \ldots, n\right\}$ of nonoverlapping closed subintervals of $\langle s, t\rangle$ will be called left-ordered (right-ordered) if $\sup I_{i+1} \leq \inf I_{i}\left(\sup I_{i}\right.$ $\left.\leq \inf I_{i+1}\right)$. Set $\Lambda-\operatorname{lovar}(x ;\langle s, t\rangle)=\sup _{1} \sum \lambda_{i} x\left(I_{i}\right)$ and $\Lambda-\operatorname{rovar}(x ;\langle s, t\rangle)=$ $\sup _{2} \sum \lambda_{i} x\left(I_{i}\right)$, where the supremum $\sup _{1}\left(\sup _{2}\right)$ is taken over all left-ordered (right-ordered) finite collections of nonoverlapping subintervals of $\langle s, t\rangle$. If $\Lambda-\operatorname{ovar}(x ;\langle\alpha, \beta\rangle)=\max \{\Lambda-\operatorname{lovar}(x ;\langle\alpha, \beta\rangle), \quad \Lambda-\operatorname{rovar}(x ;\langle\alpha, \beta\rangle)\}$ is finite, then we say $x$ is of ordered $\Lambda$-bounded variation and write $x \in O \Lambda \mathrm{BV}$. It is easy to see that the above definition is equivalent to the classical one ([4], p. 75).

Clearly, $\Lambda \mathrm{BV} \subset O \Lambda \mathrm{BV}$. In [4] D. Waterman asked: Is this inclusion proper? C..L. Belna has constructed in [1] a continuous function $f \in \mathrm{OHBV}$ such that $f \notin \mathrm{HBV}$ for the $\Lambda$-sequence $H=(1 / i)$. Since the method of $\mathrm{C}$. L. Belna is applicable only to $\mathrm{OHBV}$ and not to $O \Lambda \mathrm{BV}$ defined in the obvious way, $\mathrm{D}$. Waterman has repeated his question in [6] and in the present paper we give the positive answer in the general case.

Received by the editors August 11, 1988 and, in revised form, July 6, 1989.

1980 Mathematics Subject Classification (1985 Revision). Primary 26A45.

Key words and phrases. Function of $\Lambda$-bounded variation, function of ordered $\Lambda$-bounded variation. 
Results. Let $n, k_{1}, \ldots, k_{n}$ be positive integers and $\left(a_{i}\right)_{i=1}^{n}$ be a finite decreasing sequence of positive numbers. For a function $x:\langle\alpha, \beta\rangle \rightarrow \mathbf{R}$ we will write $x \in F_{k_{1}, \ldots, k_{n}}^{a_{1}, \ldots, a_{n}}\langle\alpha, \beta\rangle$ if there exists a finite decreasing sequence $\left(t_{i}\right)_{i=1}^{r_{n}+1}$ of points of $\langle\alpha, \beta\rangle$ such that $t_{1}=\beta, \quad t_{r_{n}+1}=\alpha$ and

$$
\begin{aligned}
& x\left(t_{i}\right)=0 \quad \text { for } i=1,3,5, \ldots, r_{n}+1 ; \\
& x\left(t_{i}\right)=a_{j} \quad \text { for } j=1, \ldots, n i=r_{j-1}+2, r_{j-1}+4, \ldots, r_{j} ; \\
& x \text { is linear on }\left\langle t_{i+1}, t_{i}\right\rangle \text { for } i=1, \ldots, r_{n},
\end{aligned}
$$

where $r_{j}=2 \sum_{i=1}^{j} k_{i} \quad$ for $j=0,1, \ldots, n$.

Lemma 1. Let $\Lambda=\left(\lambda_{i}\right)$ be a $\Lambda$-sequence and $x \in F_{k_{1}, \ldots, k_{n}}^{a_{1}, \ldots, a_{n}}\langle\alpha, \beta\rangle$. Then

$$
\Lambda-\operatorname{var}(x ;\langle\alpha, \beta\rangle)=\Lambda-\operatorname{lovar}(x ;\langle\alpha, \beta\rangle)=\sum_{j=1}^{n} a_{j} \sum_{i=r_{j-1}+1}^{r_{j}} \lambda_{i} .
$$

Proof. Setting $P_{i}=\left\langle t_{i+1}, t_{i}\right\rangle$ for $i=1, \ldots, r_{n}$, we have $\sup P_{i+1}=\inf P_{i}$. Thus

$$
\begin{aligned}
\Lambda-\operatorname{var}(x ;\langle\alpha, \beta\rangle) & \geq \Lambda-\operatorname{lovar}(x ;\langle\alpha, \beta\rangle) \\
& \geq \sum_{i=1}^{r_{n}} \lambda_{i} x\left(P_{i}\right)=\sum_{j=1}^{n} a_{j} \sum_{i=r_{j-1}+1}^{r_{j}} \lambda_{i} .
\end{aligned}
$$

Hence, it is enough to show that

$$
\Lambda-\operatorname{var}(x ;\langle\alpha, \beta\rangle) \leq \sum_{i=1}^{r_{n}} \lambda_{i} x\left(P_{i}\right) .
$$

Observe that for every finite collection $\left\{I_{i}: i=1, \ldots, s\right\}$ of nonoverlapping subintervals of $\langle\alpha, \beta\rangle$ there exists a collection $\left\{I_{i}^{\prime}: i=1, \ldots, s\right\}$ of subintervals of $\langle\alpha, \beta\rangle$ such that $I_{i}^{\prime} \subset I_{i}, \quad x\left(I_{i}^{\prime}\right)=x\left(I_{i}\right)$ and $\left\{t_{i}: i=1, \ldots, r_{n}+1\right\} \cap$ int $I_{j}^{\prime}=\varnothing$ for $j=1, \ldots, s$. Without loss of generality we may assume that the sets $Z_{i}=\left\{j=1, \ldots, s: I_{j}^{\prime} \subset\left\langle t_{i+1}, t_{i}\right\rangle\right\}$ are nonempty for $i=1, \ldots, r_{n}$. Setting $m_{i}=\min Z_{i}$ and arranging all $m_{i}$ in the decreasing order $w_{1}>\cdots>w_{r_{n}}$, we have $\lambda_{w_{i}} \leq \lambda_{i}$ for $i=1, \ldots, r_{n}$. Therefore, by ([2], Theorem 368)

$$
\begin{aligned}
\sum_{j=1}^{s} \lambda_{j} x\left(I_{j}\right) & =\sum_{i=1}^{r_{n}} \sum_{j \in Z_{i}} \lambda_{j} x\left(I_{j}^{\prime}\right) \leq \sum_{i=1}^{r_{n}} \lambda_{m_{i}} \sum_{j \in Z_{i}} x\left(I_{j}^{\prime}\right) \\
& \leq \sum_{i=1}^{r_{n}} \lambda_{m_{i}} x\left(P_{i}\right) \leq \sum_{i=1}^{r_{n}} \lambda_{w_{i}} x\left(P_{i}\right) \leq \sum_{i=1}^{r_{n}} \lambda_{i} x\left(P_{i}\right) .
\end{aligned}
$$

So by ([3], Theorem 1) the proof is complete. 
For a $\Lambda$-sequence $\Lambda=\left(\lambda_{i}\right)$, a function $x \in F_{k_{1}, \ldots, k_{n}}^{a_{1}, \ldots, a_{n}}\langle\alpha, \beta\rangle$ and $s=$ $1, \ldots, r_{n}$ we set $j_{s}=\max \left\{j=1, \ldots, n: r_{j-1}<s\right\}, \quad p_{s}=s-r_{j_{s}-1}$ and

$$
v(\Lambda, x, s)=a_{j_{s}} \sum_{i=1}^{p_{s}} \lambda_{i}+\sum_{m=1}^{j_{s}-1} a_{m} \sum_{i=p_{s}+r_{j_{s}-1}-r_{m}+1}^{p_{s}+r_{j_{s}-1}-r_{m-1}} \lambda_{i}
$$

Lemma 2. Let $\Lambda=\left(\lambda_{i}\right)$ be a $\Lambda$-sequence and $x \in F_{k_{1}, \ldots, k_{n}}^{a_{1}, \ldots, a_{n}}\langle\alpha, \beta\rangle$. Then $\Lambda$ $\operatorname{rovar}(x:\langle\alpha, \beta\rangle)=\max \left\{v(\Lambda, x, s): s=1, \ldots, r_{n}\right\}$.

Proof. Set $P_{i}=\left\langle t_{i+1}, t_{i}\right\rangle$ for $i=1, \ldots, r_{n}$. Since

$$
\begin{aligned}
\Lambda-\operatorname{rovar}(x ;\langle\alpha, \beta\rangle) & \geq \max \left\{\sum_{i=1}^{s} \lambda_{i} x\left(P_{s+1-i}\right): s=1, \ldots, r_{n}\right\} \\
& =\max \left\{v(\Lambda, x, s): s=1, \ldots, r_{n}\right\},
\end{aligned}
$$

it is enough to show that

$$
\Lambda-\operatorname{rovar}(x,\langle\alpha, \beta\rangle) \leq \max \left\{v(\Lambda, x, s): s=1, \ldots, r_{n}\right\} .
$$

Let $\left\{I_{j}: j=1, \ldots, k\right\}$ be a finite right-ordered collection of nonoverlapping subintervals of $\langle\alpha, \beta\rangle$. Without loss of generality we may assume that $\left\{t_{i}: i=\right.$ $\left.1, \ldots, r_{n}+1\right\} \cap$ int $I_{j}=\varnothing$ for $j=1, \ldots, k$. Let $Z_{i}=\left\{j=1, \ldots, k: I_{j} \subset\right.$ $\left.\left\langle t_{i+1}, t_{i}\right\rangle\right\}$ for $i=1, \ldots, r_{n}$. Setting $L=\left\{i=1, \ldots, r_{n}: Z_{i} \neq \varnothing\right\}$ and $c=$ card $L$, we have $c \leq r_{n}$. Further, set $m_{i}=\min Z_{i}$ for $i \in L$. Then $\lambda_{j} \leq \lambda_{m_{i}}$ for $j \in Z_{i}$. Arranging all elements of $L$ in the decreasing order $w_{1}>\cdots>w_{c}$, we have $m_{w_{i}} \geq i$ and $w_{i} \geq c+1-i$ for $i=1, \ldots, c$. Thus $\lambda_{m_{w_{i}}} \leq \lambda_{i}$ and $x\left(P_{w_{i}}\right) \leq x\left(P_{c+1-i}\right)$ for $i=1, \ldots, c$. Finally,

$$
\begin{aligned}
\sum_{j=1}^{k} \lambda_{j} x\left(I_{j}\right) & =\sum_{i=1}^{c} \sum_{j \in Z_{w_{i}}} \lambda_{j} x\left(I_{j}\right) \leq \sum_{i=1}^{c} \lambda_{m_{w_{i}}} \sum_{j \in Z_{w_{i}}} x\left(I_{j}\right) \\
& \leq \sum_{i=1}^{c} \lambda_{m_{w_{i}}} x\left(P_{w_{i}}\right) \leq \sum_{i=1}^{c} \lambda_{i} x\left(P_{c+1-i}\right) \\
& =v(\Lambda, x, c) \leq \max \left\{v(\Lambda, x, s): s=1, \ldots, r_{n}\right\}
\end{aligned}
$$

Lemma 3. Let $n$ be a positive integer. Then for arbitrary numbers $a, \gamma, \varepsilon>0$ and arbitrary $\Lambda$-sequence $\Lambda=\left(\lambda_{i}\right)$ there exist positive numbers $a_{1}>\cdots>a_{n}$ with $a_{1} \leq a$ and positive integers $k_{1}, \ldots, k_{n}$ such that $\Lambda-\operatorname{var}(x ;\langle\alpha, \beta\rangle)=n \gamma$ and $\Lambda-\operatorname{rovar}(x ;\langle\alpha, \beta\rangle) \leq \gamma+\varepsilon \quad$ for $x \in F_{k_{1}, \ldots, k_{n}}^{a_{1}, \ldots, a_{n}}\langle\alpha, \beta\rangle$.

Proof. Let $n=1$. Given positive numbers $a, \gamma, \varepsilon$ and a $\Lambda$-sequence $\Lambda=\left(\lambda_{i}\right)$ we set

$$
k_{1}=\min \left\{m=1,2, \ldots: a \sum_{i=1}^{2 m} \lambda_{i} \geq \gamma\right\} \quad \text { and } \quad a_{1}=\gamma\left(\sum_{i=1}^{2 k_{1}} \lambda_{i}\right)^{-1} .
$$


For $x \in F_{k_{1}}^{a_{1}}\langle\alpha, \beta\rangle$ we have by Lemma 1

$$
\Lambda-\operatorname{var}(x ;\langle\alpha, \beta\rangle)=a_{1} \sum_{i=1}^{2 k_{1}} \lambda_{i}=\gamma
$$

and by Lemma 2

$$
\Lambda-\operatorname{rovar}(x ;\langle\alpha, \beta\rangle) \leq \max \left\{a_{1} \sum_{i=1}^{s} \lambda_{i}: s=1, \ldots, 2 k_{1}\right\}=\gamma<\gamma+\varepsilon
$$

Now assume that $n$ is a positive integer such that for every positive number $a, \gamma, \varepsilon$ and every $\Lambda$-sequence $\Lambda$ there exist positive numbers $a_{1}>\cdots>a_{n}$ with $a_{1} \leq a$ and positive integers $k_{1}, \ldots, k_{n}$ such that $\Lambda-\operatorname{var}(x ;\langle\alpha, \beta\rangle)=n \gamma$ and $\Lambda-\operatorname{rovar}(x ;\langle\alpha, \beta\rangle) \leq \gamma+\varepsilon$ for $x \in F_{k_{1}, \ldots, k_{n}}^{a_{1}, \ldots, a_{n}}\langle\alpha, \beta\rangle$. It is easy to see

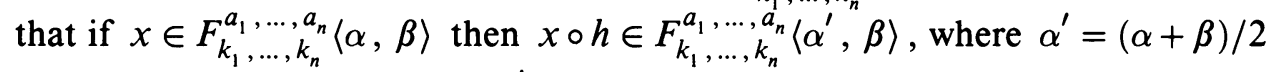
and $h(t)=2 t-\beta$ for $t \in\left\langle\alpha^{\prime}, \beta\right\rangle$. Hence, given positive numbers $a, \gamma, \varepsilon$ and a $\Lambda$-sequence $\Lambda=\left(\lambda_{i}\right)$, there exist positive numbers $a_{1}>\cdots>a_{n}$ with $a_{1} \leq a$ and positive integers $k_{1}, \ldots, k_{n}$ such that $\Lambda-\operatorname{var}\left(x ;\left\langle\alpha^{\prime}, \beta\right\rangle\right)=n \gamma$ and $\Lambda-\operatorname{rovar}\left(x ;\left\langle\alpha^{\prime}, \beta\right\rangle\right) \leq \gamma+\varepsilon / 2$ for $x \in F_{k_{1}, \ldots, k_{n}}^{a_{1}, \ldots, a_{n}}\left\langle\alpha^{\prime}, \beta\right\rangle$. Set $k=\sum_{i=1}^{n} k_{i}$ and

$$
\begin{gathered}
(+) \quad i_{0}=\min \left\{i=1,2, \ldots: \frac{\lambda_{2 k+i}}{\lambda_{2 k}} \leq \frac{\varepsilon}{4 \gamma+2 \varepsilon}\right\} \\
(++) d=\min \left\{\frac{a_{n}}{2}, \varepsilon\left(4 \sum_{i=1}^{2 k} \lambda_{i}\right)^{-1}, \varepsilon\left(4 \sum_{i=2 k+1}^{2 k+i_{0}} \lambda_{i}\right)^{-1}\right\} \\
k_{n+1}=\min \left\{m=1,2, \ldots: \sum_{i=2 k+1}^{2 k+2 m} \lambda_{i} \geq \frac{\gamma}{d}\right\} \\
a_{n+1}=\gamma\left(\sum_{i=2 k+1}^{2 k+2 k_{n+1}} \lambda_{i}\right)^{-1}
\end{gathered}
$$

Then $a_{n+1} \leq d<a_{n}$ and $\Lambda_{(2 k)}-\operatorname{var}\left(x ;\left\langle\alpha, \alpha^{\prime}\right\rangle\right)=\gamma$ for $x \in F_{k_{n+1}}^{a_{n+1}}\left\langle\alpha, \alpha^{\prime}\right\rangle$. Given functions $\left.x_{1} \in F_{k_{1}, \ldots, k_{n}}^{a_{1}, \ldots, a_{n}}, \beta\right\rangle, x_{2} \in F_{k_{n+1}}^{a_{n+1}}\left\langle\alpha, \alpha^{\prime}\right\rangle$, we set

$$
y(t)= \begin{cases}x_{1}(t) & \text { for } t \in\left(\alpha^{\prime}, \beta\right\rangle \\ x_{2}(t) & \text { for } t \in\left\langle\alpha, \alpha^{\prime}\right\rangle\end{cases}
$$


Then $y \in F_{k_{1}, \ldots, k_{n+1}}^{a_{1}, \ldots, a_{n+1}}\langle\alpha, \beta\rangle$ and by Lemma 1

$$
\begin{aligned}
\Lambda-\operatorname{var}(y ;\langle\alpha, \beta\rangle) & =\sum_{j=1}^{n+1} a_{j} \sum_{i=r_{j-1}+1}^{r_{j}} \lambda_{i} \\
& =a_{n+1} \sum_{i=2 k+1}^{2 k+2 k_{n+1}} \lambda_{i}+\sum_{j=1}^{n} a_{j} \sum_{i=r_{j-1}+1}^{r_{j}} \lambda_{i} \\
& =\Lambda_{(2 k)}-\operatorname{var}\left(x_{2} ;\left\langle\alpha, \alpha^{\prime}\right\rangle\right)+\Lambda-\operatorname{var}\left(x_{1} ;\left\langle\alpha^{\prime}, \beta\right\rangle\right) \\
& =(n+1) \gamma .
\end{aligned}
$$

For $s=1, \ldots, r_{n}$ we have

(a) if $1 \leq s \leq 2 k=r_{n}$ then

$$
v(\Lambda, y, s)=v\left(\Lambda, x_{1}, s\right) \leq \Lambda-\operatorname{rovar}\left(x_{1} ;\left\langle\alpha^{\prime}, \beta\right\rangle\right) \leq \gamma+\varepsilon / 2
$$

(b) if $2 k+1 \leq s$ then

$\left(\mathrm{b}_{1}\right)$ if $p_{s} \leq 2 k+i_{0}$ then by $(++)$ and by (a)

$$
\begin{aligned}
v(\Lambda, y, s) & =a_{n+1} \sum_{i=1}^{p_{s}} \lambda_{i}+\sum_{m=1}^{n} a_{m} \sum_{i=p_{s}+r_{n}-r_{m}+1}^{p_{s}+r_{n}-r_{m-1}} \lambda_{i} \\
& \leq a_{n+1} \sum_{i=1}^{2 k} \lambda_{i}+a_{n+1} \sum_{i=2 k+1}^{2 k+i_{0}} \lambda_{i}+\sum_{m=1}^{n} a_{m} \sum_{i=r_{n}-r_{m}+1}^{r_{n}-r_{m-1}} \lambda_{p_{s}+i} \\
& \leq \frac{\varepsilon}{4}+\frac{\varepsilon}{4}+\sum_{m=1}^{n} a_{m} \sum_{i=r_{n}-r_{m}+1}^{r_{n}-r_{m-1}} \lambda_{i} \\
& =\frac{\varepsilon}{2}+v(\Lambda, y, 2 k) \leq \gamma+\varepsilon ;
\end{aligned}
$$

$\left(\mathrm{b}_{2}\right)$ if $p_{s}>2 k+i_{0}$ then by $(+)$

$$
\frac{\lambda_{p_{s}+i}}{\lambda_{1}} \leq \frac{\lambda_{2 k+i_{0}}}{\lambda_{2 k}} \leq \frac{\varepsilon}{4 \gamma+2 \varepsilon} \quad \text { for } i=1, \ldots, 2 k
$$

and thus, by (a),

$$
\begin{aligned}
v(\Lambda, y, s) & =a_{n+1} \sum_{i=1}^{2 k} \lambda_{i}+a_{n+1} \sum_{i=2 k+1}^{p_{s}} \lambda_{i}+\sum_{m=1}^{n} a_{m} \sum_{i=r_{n}-r_{m}+1}^{r_{n}-r_{m-1}} \lambda_{p_{s}+i} \\
& \leq \frac{\varepsilon}{4}+a_{n+1} \sum_{i=2 k+1}^{2 k+2 k_{n+1}} \lambda_{i}+\sum_{m=1}^{n} a_{m} \sum_{i=r_{n}-r_{m}+1}^{r_{n}-r_{m-1}} \lambda_{2 k+i_{0}+i} \\
& \leq \frac{\varepsilon}{4}+\Lambda_{(2 k)}-\operatorname{var}\left(x_{2} ;\left\langle\alpha, \alpha^{\prime}\right\rangle\right)+\sum_{m=1}^{n} a_{m} \sum_{i=r_{n}-r_{m}+1}^{r_{n}-r_{m-1}} \frac{\lambda_{i} \varepsilon}{4 \gamma+2 \varepsilon} \\
& =\frac{\varepsilon}{4}+\gamma+\frac{\varepsilon}{4 \gamma+2 \varepsilon} v(\Lambda, y, 2 k) .
\end{aligned}
$$


Therefore, by Lemma $2, \Lambda-\operatorname{rovar}(y ;\langle\alpha, \beta\rangle) \leq \gamma+\varepsilon$. Since for every $x \in$ $F_{k_{1}, \ldots, k_{n+1}}^{a_{1}, \ldots, a_{n+1}}\langle\alpha, \beta\rangle$ we have $\Lambda-\operatorname{var}(x ;\langle\alpha, \beta\rangle)=\Lambda-\operatorname{var}(y ;\langle\alpha, \beta\rangle)$ and $\Lambda-$ $\operatorname{rovar}(x ;\langle\alpha, \beta\rangle)=\Lambda-\operatorname{rovar}(y ;\langle\alpha, \beta\rangle)$, the proof is complete.

Theorem. For every $\Lambda$-sequence $\Lambda=\left(\lambda_{i}\right)$ there exists a continuous $x \in O \Lambda \mathrm{BV}-$ $\Lambda \mathrm{BV}$.

Proof. Let $\left(t_{n}\right)$ be an increasing convergent-to- $\beta$ sequence of points of $\langle\alpha, \beta\rangle$ with $t_{1}=\alpha$ and let $\sum \varepsilon_{i}$ be a convergent series of positive numbers. By Lemmas 3 and 1 for $n=1,2, \ldots$ there exist positive numbers $a_{1}^{n}>\cdots>a_{2^{n}}^{n}$ with

$$
a_{1}^{n} \leq d_{n},
$$

positive integers $k_{1}^{n}, \ldots, k_{2^{n}}^{n}$ and functions $x_{n} \in F_{k_{1}^{n}, \ldots, k_{2^{n}}^{n}}^{a_{n}^{n}}\left\langle t_{n}, t_{n+1}\right\rangle$ such that

$$
\Lambda_{\left(w_{n-1}\right)}-\operatorname{var}\left(x_{n} ;\left\langle t_{n}, t_{n+1}\right\rangle\right)=\Lambda_{\left(w_{n-1}\right)}-\operatorname{lovar}\left(x_{n} ;\left\langle t_{n}, t_{n+1}\right\rangle\right)=2^{n} 2^{-n}
$$

and

$$
\Lambda_{\left(w_{n-1}\right)}-\operatorname{rovar}\left(x_{n} ;\left\langle t_{n}, t_{n+1}\right\rangle\right) \leq 2^{-n}+\varepsilon_{n} / 2
$$

for $n=1,2, \ldots$, where

$$
\begin{gathered}
d_{1}=1 \quad \text { and for } n=1,2, \ldots \\
d_{n+1}=\min \left\{\frac{a_{2^{n}}^{n}}{2}, \varepsilon_{n+1}\left(2 \sum_{i=1}^{w_{n}} \lambda_{i}\right)^{-1}, \quad \varepsilon_{n+1}\left(2 \sum_{i=w_{n}+1}^{w_{n}+v_{n+1}} \lambda_{i}\right)^{-1}\right\} \\
w_{n}=2 \sum_{j=1}^{n} \sum_{i=1}^{2^{j}} k_{i}^{j} \quad \text { for } n=0,1,2, \ldots
\end{gathered}
$$

(6) $v_{n+1}=\min \left\{i=1,2, \ldots: 4 \sum_{j=1}^{n} \sum_{i=1}^{2^{j}} k_{i}^{j} a_{i}^{j}<\frac{\varepsilon_{n+1}}{\lambda_{w_{n}+i}}\right\} \quad$ for $n=1,2, \ldots$

For $n=1,2, \ldots$ we define a continuous function $y_{n}:\left\langle t_{1}, t_{n+1}\right\rangle \rightarrow \mathbf{R}$ by $y_{n}(t)=x_{i}(t)$ for $t \in\left\langle t_{i}, t_{i+1}\right\rangle, \quad i=1, \ldots, n$. We shall show that for $n=$ $1,2, \ldots$ the following inequality holds:

$$
\Lambda-\operatorname{lovar}\left(y_{n} ;\left\langle t_{1}, t_{n+1}\right\rangle\right) \leq 1+\sum_{i=1}^{n} \varepsilon_{i} .
$$

By (2) it is clear for $n=1$, because $x_{1}=y_{1}$. Assume that $n$ is a positive integer such that (7) holds. Let $\left\{I_{i}: i=1, \ldots, l\right\}$ be a finite left-ordered collection of nonoverlapping subintervals of $\left\langle t_{1}, t_{n+2}\right\rangle$. Without loss of generality we may assume $t_{n+1} \notin$ int $I_{i}$ for $i=1, \ldots, l$. Set $z=\max \left\{i=1, \ldots, l: I_{i} \subset\right.$ $\left.\left\langle t_{n+1}, t_{n+2}\right\rangle\right\}$ and $h=\min \left\{z, w_{n}\right\}$. If $z \leq w_{n}+v_{n+1}$ then we have by the 
inductional assumption and by (1), (4)

$$
\begin{aligned}
\sum_{i=1}^{l} \lambda_{i} y_{n+1}\left(I_{i}\right) & \leq \sum_{i=1}^{h} \lambda_{i} x_{n+1}\left(I_{i}\right)+\sum_{i=w_{n}+1}^{z} \lambda_{i} x_{n+1}\left(I_{i}\right)+\sum_{i=z+1}^{l} \lambda_{i} y_{n}\left(I_{i}\right) \\
& \leq \sum_{i=1}^{h} \lambda_{i} a_{1}^{n+1}+\sum_{i=w_{n}+1}^{w_{n}+v_{n+1}} \lambda_{i} a_{1}^{n+1}+\sum_{i=z+1}^{l} \lambda_{i-z} y_{n}\left(I_{i}\right) \\
& \leq \frac{\varepsilon_{n+1}}{2}+\frac{\varepsilon_{n+1}}{2}+\Lambda-\operatorname{lovar}\left(y_{n} ;\left\langle t_{1}, t_{n+1}\right\rangle\right) \\
& \leq 1+\sum_{i=1}^{n+1} \varepsilon_{i} .
\end{aligned}
$$

If $z>w_{n}+v_{n+1}$ then by (6)

$$
\begin{aligned}
\sum_{i=1+z}^{l} \lambda_{i} y_{n+1}\left(I_{i}\right) & \leq \lambda_{z+1} \sum_{i=z+1}^{l} y_{n}\left(I_{i}\right) \\
& \leq 2 \lambda_{w_{n}+v_{n+1}} \sum_{j=1}^{n} \sum_{i=1}^{2^{j}} k_{i}^{j} a_{i}^{j} \leq \frac{\varepsilon_{n+1}}{2}
\end{aligned}
$$

and by (1), (4), (2)

$$
\begin{aligned}
\sum_{i=1}^{z} \lambda_{i} y_{n+1}\left(I_{i}\right) & \leq \sum_{i=1}^{w_{n}} \lambda_{i} x_{n+1}\left(I_{i}\right)+\sum_{i=w_{n}+1}^{z} \lambda_{i} x_{n+1}\left(I_{i}\right) \\
& \leq a_{1}^{n+1} \sum_{i=1}^{w_{n}} \lambda_{i}+\Lambda_{\left(w_{n}\right)}-\operatorname{var}\left(x_{n+1} ;\left\langle t_{n+1}, t_{n+2}\right\rangle\right) \\
& \leq \frac{\varepsilon_{n+1}}{2}+1 .
\end{aligned}
$$

Therefore,

$$
\Lambda-\operatorname{lovar}\left(y_{n+1} ;\left\langle t_{1}, t_{n+2}\right\rangle\right) \leq 1+\sum_{i=1}^{n+1} \varepsilon_{i} .
$$

Now, we shall show that

$$
\Lambda-\operatorname{rovar}\left(y_{n} ;\left\langle t_{1}, t_{n+1}\right\rangle\right) \leq \sum_{i=1}^{n} 2^{-i}+\sum_{i=1}^{n} \varepsilon_{i}
$$

for $n=1,2, \ldots$. For $n=1$ it is clear by (2). Assume that $n$ is a positive integer such that (8) holds. Let $\left\{I_{i}: i=1, \ldots, l\right\}$ be a finite right-ordered collection of nonoverlapping subintervals of $\left\langle t_{1}, t_{n+2}\right\rangle$. Without loss of generality we may assume that $t_{n+1} \notin$ int $I_{i}$ for $i=1, \ldots, l$. Setting $z=$ $\max \left\{i=1, \ldots, l: I_{i} \subset\left\langle t_{1}, t_{n+1}\right\rangle\right\}(\max \varnothing=0)$ and $h=\min \left\{l, \max \left\{x, w_{n}\right\}\right\}$, 
we have by (1), (4), (3) and by the inductional assumption

$$
\begin{aligned}
& \sum_{i=1}^{l} \lambda_{i} y_{n+1}\left(I_{i}\right) \\
& \quad \leq \sum_{i=1}^{z} \lambda_{i} y_{n}\left(I_{i}\right)+\sum_{i=z+1}^{h} \lambda_{i} x_{n+1}\left(I_{i}\right)+\sum_{i=h+1}^{l} \lambda_{i} x_{n+1}\left(I_{i}\right) \\
& \quad \leq \Lambda-\operatorname{rovar}\left(y_{n} ;\left\langle t_{1}, t_{n+1}\right\rangle\right)+a_{1}^{n+1} \sum_{i=1}^{w_{n}} \lambda_{i}+\Lambda_{\left(w_{n}\right)}-\operatorname{var}\left(x_{n+1} ;\left\langle t_{n+1}, t_{n+2}\right\rangle\right) \\
& \quad \leq \sum_{i=1}^{n} 2^{-i}+\sum_{i=1}^{n} \varepsilon_{i}+\frac{\varepsilon_{n+1}}{2}+2^{-n-1}+\frac{\varepsilon_{n+1}}{2}
\end{aligned}
$$

Therefore,

$$
\Lambda-\operatorname{rovar}\left(y_{n+1} ;\left\langle t_{1}, t_{n+2}\right\rangle\right) \leq \sum_{i=1}^{n+1} 2^{-i}+\sum_{i=1}^{n+1} \varepsilon_{i} .
$$

Finally, we define the function $y:\langle\alpha, \beta\rangle \rightarrow \mathbf{R}$ by $y(\beta)=0$ and $y(t)=$ $x_{n}(t)$ for $t \in\left\langle t_{n}, t_{n+1}\right), \quad n=1,2, \ldots$. The function $y$ is continuous, since $a_{1}^{n} \leq 2^{-n+1}$ for $n=1,2, \ldots$. It is easy to see that $\Lambda-\operatorname{var}(y ;\langle\alpha, \beta\rangle) \geq$ $\sum_{n} \Lambda_{\left(w_{n-1}\right)}-\operatorname{var}\left(x_{n} ;\left\langle t_{n}, t_{n+1}\right\rangle\right)$. Thus, by (2) $x$ is not of $\Lambda$-bounded variation.

Observe that for every finite left-ordered collection $\left\{I_{i}: i=1, \ldots, l\right\}$ of nonoverlapping subintervals of $\langle\alpha, \beta\rangle$ there exists a positive integer $n$ and a finite left-ordered collection $\left\{I_{i}^{\prime}: i=1, \ldots, l\right\}$ such that $I_{i}^{\prime} \subset I_{i}, \quad x\left(I_{i}^{\prime}\right)=$ $x\left(I_{i}\right)$ and $\sup I_{1}^{\prime} \leq t_{n+1}$. Thus

$$
\sum_{i=1}^{l} \lambda_{i} y\left(I_{i}\right) \leq \Lambda-\operatorname{lovar}\left(y_{n} ;\left\langle t_{1}, t_{n+1}\right\rangle\right)
$$

and by (7) we have

$$
\Lambda-\operatorname{lovar}(y ;\langle\alpha ; \beta\rangle) \leq 1+\sum_{i} \varepsilon_{i}
$$

It may be proved in a similar manner that

$$
\Lambda-\operatorname{rovar}(y ;\langle\alpha, \beta\rangle) \leq 1+\sum_{i} \varepsilon_{i}
$$

This fact together with (9) implies that $y \in O \Lambda \mathrm{BV}$.

The remarks from $\S \S 2$ and 3 of [5] are true for arbitrary $\Lambda$-sequence $\Lambda$. In particular, $O \Lambda \mathrm{BV}$ is a Banach space with norm

$$
\|g\|=|g(\alpha)|+\Lambda-\operatorname{ovar}(g ;\langle\alpha, \beta\rangle)
$$


and one has

Corollary. $\Lambda \mathrm{BV}$ is a first category subset of $\mathrm{O} \Lambda \mathrm{BV}$ for arbitrary $\Lambda$-sequence $\Lambda$.

Observe that the knowledge of construction of $x \in O \Lambda \mathrm{BV}-\Lambda \mathrm{BV}$ is not necessary to prove the above corollary. Namely, let $x \in O \Lambda \mathrm{BV}-\Lambda \mathrm{BV}$. Then there exists a sequence $\left\{I_{n}\right\}$ of nonoverlapping subintervals of $\langle\alpha, \beta\rangle$ such that $\sum \lambda_{n} x\left(I_{n}\right)=\infty$. It follows that there exists a subsequence $\left\{I_{n_{i}}\right\}$ such that $r\left(x, I_{n_{i}}\right)>0$ and $\sum \lambda_{i} r\left(x, I_{n_{i}}\right)=\sum \lambda_{i} x\left(I_{n_{i}}\right)=\infty$ (we replace $x$ by $-x$, if necessary). Set

$$
m_{k}=\min \left\{m=1,2, \ldots: \sum_{i=1}^{m} \lambda_{i} r\left(x, I_{n_{i}}\right) \geq k\right\}
$$

and

$$
F_{k}(g)=\sum_{i=1}^{m_{k}} \lambda_{i} r\left(g, I_{n_{i}}\right)
$$

for $k=1,2, \ldots ; g \in O \Lambda \mathrm{BV}$. It is easy to see that for $k=1,2, \ldots, \quad F_{k}$ is a continuous linear functional on $O \Lambda \mathrm{BV}$. Using D. Waterman's arguments ([5], proof of Proposition 3), we complete the proof.

\section{REFERENCES}

1. C. L. Belna, On ordered harmonic bounded variation, Proc. Amer. Math. Soc. 80 (1980), 441-444.

2. G. H. Hardy, J. E. Littlewood, and G. Pòlya, Inequalities, 2nd ed., Cambridge University Press, 1964.

3. D. Waterman, On $\Lambda$-bounded variation, Studia Math. 57 (1976), 33-45.

4. $\ldots, \Lambda$-bounded variation: recent results and unsolved problems, Real Anal. Exchange 4 (1978-79), 69-75.

5. __ On the note of C. L. Belna, Proc. Amer. Math. Soc. 80 (1980), 445-447.

6. __ Generalized bounded variation-recent results and open questions, Real Anal. Exchange 5 (1979-80), 148-150.

Institute of Mathematics, University of SzCzecin, Ul. Wielkopolska 15, 70-451

SzCZeCin, Poland 